\title{
Polyunsaturated fatty acids and risk of Alzheimer's disease: a Mendelian randomization study
}

\author{
Yasutake Tomata $^{1,2}$ (D) Susanna C. Larsson ${ }^{3} \cdot$ Sara Hägg $^{1}$
}

Received: 23 July 2019 / Accepted: 21 October 2019 / Published online: 1 November 2019

(c) The Author(s) 2019

\begin{abstract}
Purpose Observational studies have suggested that polyunsaturated fatty acids (PUFAs) may decrease Alzheimer's disease (AD) risk. In the present study, we examined this hypothesis using a Mendelian randomization analysis.

Methods We used summary statistics data for single-nucleotide polymorphisms associated with plasma levels of n-6 PUFAs (linoleic acid, arachidonic acid) and n-3 PUFAs (alpha-linolenic acid, eicosapentaenoic acid, docosapentaenoic acid, docosahexaenoic acid), and the corresponding data for $\mathrm{AD}$ from a genome-wide association meta-analysis of 63,926 individuals (21,982 diagnosed AD cases, 41,944 controls).

Results None of the genetically predicted PUFAs was significantly associated with AD risk; odds ratios (95\% confidence interval) per 1 SD increase in PUFA levels were $0.98(0.93,1.03)$ for linoleic acid, $1.01(0.98,1.05)$ for arachidonic acid, $0.96(0.88,1.06)$ for alpha-linolenic acid, $1.03(0.93,1.13)$ for eicosapentaenoic acid, $1.03(0.97,1.09)$ for docosapentaenoic acid, and $1.01(0.81,1.25)$ for docosahexaenoic acid.
\end{abstract}

Conclusions This study did not support the hypothesis that PUFAs decrease AD risk.

Keywords Polyunsaturated fatty acids · Alzheimer's disease $\cdot$ Mendelian randomization analysis

$\begin{array}{ll}\text { Abbreviations } \\ \text { PUFA } & \text { Polyunsaturated fatty acid } \\ \text { AD } & \text { Alzheimer's disease } \\ \text { RCT } & \text { Randomized controlled trial } \\ \text { MR } & \text { Mendelian randomization } \\ \text { LA } & \text { Linoleic acid } \\ \text { AA } & \text { Arachidonic acid } \\ \text { ALA } & \text { Alpha-linolenic acid } \\ \text { EPA } & \text { Eicosapentaenoic acid } \\ \text { DPA } & \text { Docosapentaenoic acid }\end{array}$

Electronic supplementary material The online version of this article (https://doi.org/10.1007/s00394-019-02126-x) contains supplementary material, which is available to authorized users.

Yasutake Tomata yasutake.tomata@ki.se

1 Department of Medical Epidemiology and Biostatistics, Karolinska Institute, Nobels väg 12A, 17156 Stockholm, Sweden

2 Division of Epidemiology, Department of Health Informatics and Public Health, Tohoku University School of Public Health, Graduate School of Medicine, Sendai, Japan

3 Department of Surgical Sciences, Uppsala University, Uppsala, Sweden

\section{DHA Docosahexaenoic acid \\ GWAS Genome-wide association study}

\section{Introduction}

The overall results from observational studies suggest that daily intake of polyunsaturated fatty acids (PUFAs) is inversely associated with risk of incident Alzheimer's disease (AD) and cognitive impairment [1]. Previous prospective studies of PUFA biomarkers have also supported the preventable association [2,3].

However, observational studies are generally susceptible to methodological problems such as confounding. Therefore, randomized controlled trials (RCTs) are more suitable to examine causal relationships. However, no RCT has yet reported the preventive effect of PUFAs on incident AD among cognitively normal adults [4].

To overcome the problem of confounding in observational studies, Mendelian randomization (MR) is becoming widespread for assessing causal relationships. MR study is a type of instrumental variable analysis where genetic variants (single-nucleotide polymorphisms: SNPs) are applied as instrumental variables for the potential risk factor. MR 
study is often described as a "natural RCT" because random allocation of alleles during meiosis is conceptually similar to the RCT design. Therefore, a MR study would provide more robust evidence regarding the causal relationship between PUFAs and AD than observational studies. However, to our knowledge, no MR study has yet investigated this relationship.

The aim of the present MR study was to examine the hypothesis that higher plasma levels of PUFAs decrease AD risk. We examined the main six types of PUFAs, including the n-6 PUFAs linoleic acid (LA) and arachidonic acid (AA) and the n-3 PUFAs alpha-linolenic acid (ALA), eicosapentaenoic acid (EPA), docosapentaenoic acid (DPA), and docosahexaenoic acid (DHA).

\section{Methods}

\section{Study design}

To estimate causal associations between lifelong exposures to PUFA levels and the risk of late-onset AD, we conducted a two-sample MR analysis using summary statistics data from genome-wide association studies (GWAS).

\section{Summary statistics data for PUFAs}

We used summary statistics data from the Cohorts for Heart and Aging Research in Genomic Epidemiology (CHARGE) consortium examining the association between genetic variants and plasma levels of n-6 PUFAs [5] and n-3 PUFAs [6]. These summary statistics were based on a meta-analysis of GWAS of individuals of European ancestry $(n=8631$ individuals for $\mathrm{n}-6$ PUFAs and 8866 individuals for $\mathrm{n}-3$ PUFAs). Age ranged from 21 to 102 years [6].

\section{Summary statistics data for AD}

We used two types of summary statistics data for AD.

First, to conduct primary analysis, we used data from GWAS for the association between genetic variants and clinically diagnosed late-onset AD (Table S1) [7]. The summary statistics data was based on a meta-analysis of GWAS from four consortia comprising a total of 63,926 individuals of European ancestry (21,982 AD cases and 41,944 cognitively normal controls). The mean age at $\mathrm{AD}$ onset ranged from 71.1 to 82.6 years [7].

Second, to conduct secondary analysis, we used summary statistics data from another GWAS for the association between genetic variants and $\mathrm{AD}$ including $\mathrm{AD}$-by-proxy based on parental diagnoses (Table S2) [8]. The summary statistics data was based on a meta-analysis consisting of a total of 455,258 individuals of European ancestry $(71,880 \mathrm{AD}$ cases, 383,378 controls). The AD-by-proxy phenotype showed a strong genetic correlation with $\mathrm{AD}\left(r_{\mathrm{g}}=0.81\right)$ [8].

\section{Selection of instrumental variables}

We selected 8 SNPs associated with one or more of the PUFAs (Table S1). All SNPs satisfied the following conditions: (1) associated with the PUFAs at genome-wide significance $\left(P<5 \times 10^{-8}\right)$; (2) biologically relevant to PUFAs (involved in PUFA metabolism); (3) strongest association within the specific locus (e.g., among $F A D S 1$ ) [5, 6]. In addition, the same SNPs have been used as instrumental variables for PUFAs in previous MR studies, and were not considered as weak instrumental variables $(F$ statistic $\geq 10)[9]$.

\section{Mendelian randomization analysis}

For each PUFA, two-sample MR analyses were performed to calculate the odds ratios and $95 \%$ confidence intervals for AD. Odds ratios were shown as per one standard deviation increase based on standard deviations of the largest study (the Atherosclerosis Risk in Communities Study) in the CHARGE consortium (Table S3) $[6,10]$. All MR analyses were performed by using the "MendelianRandomization" package in R 3.5 (R Project for Statistical Computing). We mainly used the command for the inverse variance weighted method " $\mathrm{mr}$ ivw". In addition, we also conducted sensitivity analyses using the weighted median method and the MR-Egger regression method for PUFAs for which at least 3 SNPs were available as instrumental variables (i.e., LA and DPA). The weighted median approach provides a consistent estimate for a causal effect if at least $50 \%$ of the information in the analysis comes from variants that are valid instrumental variables. The MREgger regression method estimates the effect size by adjusting for horizontal pleiotropy. We tested for pleiotropy using the MR-Egger regression intercept. This test is based on the assumption that the intercept (the gene-outcome association) should be zero if the gene-exposure association is zero.

\section{Ethical issues}

Because the present study is based on GWAS summary statistics rather than individual level data, an ethical approval is not required according to the rules at Karolinska Institutet, Sweden.

\section{Results}

The results of the MR analyses based on the inverse variance weighted method are shown in Fig. 1. All genetically predicted plasma PUFA levels were not statistically significantly associated with $\mathrm{AD}$ risk. 
Results obtained by the weighted median and MR-Egger regression analyses (Table S4) were not essentially different from those based on the inverse variance weighted method (Fig. 1). Plots to visualize the results of the analyses based on the MR-Egger method are shown in Figure S1 and Figure $\mathrm{S} 2$. There was no evidence for pleiotropy based on the MREgger regression analyses $(P$-intercept $>0.05)$.

Results from the secondary analyses (Table S5) were consistent with the main findings (Fig. 1).

\section{Discussion}

To our knowledge, this is the first MR study to examine the relationship between genetically predicted levels of PUFAs and AD. Our results showed that none of the PUFAs was statistically significantly associated with AD risk. Results from our MR study, which is less prone to confounding compared with observational studies, did not support previous results from observational studies showing that PUFAs are inversely associated with AD risk.

As major methodological issues about bias towards the null in MR studies, weak instrument bias and low statistical power are often suggested. Although we only used 1-3 SNPs as instrumental variables for each PUFA, the SNPs explained a relatively large variation in PUFA levels and they fulfilled the criterion as not being weak instrumental variables ( $F$ statistic $>10)$ [9]. Additionally, to increase statistical power, we conducted secondary analyses based on a larger dataset including AD-by-proxy based on parental diagnoses (Table S5). Results of these analyses (Table S5) were consistent with the results of the main analysis (Fig. 1). Therefore, weak instrument bias and low statistical power are unlikely to explain our main results.
Regardless of statistical power, effect sizes (point estimates) were smaller in the current MR study than in previous observational studies. For example, a recent cohort study reported that the hazard ratio per 1 SD increment of red blood cell EPA + DHA levels for dementia was 0.92 $(P<0.05)$ [3]. Furthermore, results of a meta-analysis of cohort studies showed that a $0.1-\mathrm{g} /$ day increment of dietary DHA intake was significantly associated with a lower risk of $\mathrm{AD}$ (relative risk $=0.63, P<0.001$ ) [1]. However, a subgroup analysis suggested that other dietary factors (e.g., vitamin E intake) were regarded as residual confounding factors [1]. Therefore, the difference in results between the present MR study and previous observational studies might be explained by residual confounding rather than methodological issues in the MR design such as weak instrument bias.

The present study has several limitations. First, we only used 1-3 SNPs as instrumental variables for each PUFA. Therefore, regression estimates such as pleiotropy test by MR-Egger regression might not be robust. Second, because shared SNPs were used among the PUFAs (e.g., rs174547 was used for LA, AA, ALA, and DPA), identifying the effects for individual PUFAs was difficult.

In conclusion, the present MR study did not support the hypothesis that PUFAs decrease AD risk.

Acknowledgements Open access funding provided by Karolinska Institute. We would like to thank Juulia Jylhävä, Xiaoying Kang, Yunzhang Wang, and Ichiro Tsuji for their technical assistance. This work was supported by the Swedish Council for Working Life and Social Research (FORTE) (2013-2292), the Swedish Research Council (201503255), and Leading Young Researcher Overseas Visit Program from Tohoku University, Japan.
Fig. 1 Mendelian randomization results: phospholipid levels of polyunsaturated fatty acids and Alzheimer's disease (21,982 clinically diagnosed cases and 41,944 controls)
Polyunsaturated fatty acid

Linoleic acid (LA, 18:2n6)

Arachidonic acid (AA, 20:4n6)

a-Linolenic acid (ALA, 18:3n3)

Eicosapentaenoic acid (EPA, 20:5n3)

Docosapentaenoic acid (DPA, 22:5n3)

Docosahexaenoic acid (DHA, 22:6n3)

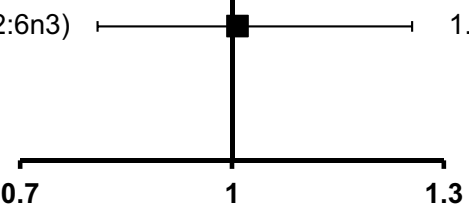

Odds Ratio of 1 SD increase in plasma PUFA levels

\begin{tabular}{ccc} 
OR & $(95 \% \mathrm{Cl})$ & P value \\
\hline 0.98 & $(0.93,1.03)$ & 0.476 \\
1.01 & $(0.98,1.05)$ & 0.471 \\
0.96 & $(0.88,1.06)$ & 0.427 \\
1.03 & $(0.93,1.13)$ & 0.610 \\
1.03 & $(0.97,1.09)$ & 0.375 \\
1.01 & $(0.81,1.25)$ & 0.944
\end{tabular}

.3 


\section{Compliance with ethical standards}

Conflict of interest All authors did not have any potential conflicts of interest related to the manuscript.

Open Access This article is distributed under the terms of the Creative Commons Attribution 4.0 International License (http://creativeco mmons.org/licenses/by/4.0/), which permits unrestricted use, distribution, and reproduction in any medium, provided you give appropriate credit to the original author(s) and the source, provide a link to the Creative Commons license, and indicate if changes were made.

\section{References}

1. Zhang Y, Chen J, Qiu J et al (2016) Intakes of fish and polyunsaturated fatty acids and mild-to-severe cognitive impairment risks: a dose-response meta-analysis of 21 cohort studies. Am J Clin Nutr 103(2):330-340. https://doi.org/10.3945/ajcn.115.124081

2. Schaefer EJ, Bongard V, Beiser AS et al (2006) Plasma phosphatidylcholine docosahexaenoic acid content and risk of dementia and Alzheimer disease: the Framingham Heart Study. Arch Neurol 63(11):1545-1550. https://doi.org/10.1001/archneur.63.11.1545

3. Ammann EM, Pottala JV, Robinson JG et al (2017) Erythrocyte omega-3 fatty acids are inversely associated with incident dementia: secondary analyses of longitudinal data from the Women's Health Initiative Memory Study (WHIMS). Prostaglandins Leukot Essent Fatty Acids 121:68-75. https://doi.org/10.1016/j.plefa .2017 .06 .006
4. Butler M, Nelson VA, Davila H et al (2018) Over-the-counter supplement interventions to prevent cognitive decline, mild cognitive impairment, and clinical Alzheimer-type dementia: a systematic review. Ann Intern Med 168(1):52-62. https://doi.org/10.7326/ m17-1530

5. Guan W, Steffen BT, Lemaitre RN et al (2014) Genome-wide association study of plasma N6 polyunsaturated fatty acids within the cohorts for heart and aging research in genomic epidemiology consortium. Circ Cardiovasc Genet 7(3):321-331. https://doi. org/10.1161/circgenetics.113.000208

6. Lemaitre RN, Tanaka T, Tang W et al (2011) Genetic loci associated with plasma phospholipid n-3 fatty acids: a meta-analysis of genome-wide association studies from the CHARGE Consortium. PLoS Genet 7(7):e1002193. https://doi.org/10.1371/journ al.pgen. 1002193

7. Kunkle BW, Grenier-Boley B, Sims R et al (2019) Genetic metaanalysis of diagnosed Alzheimer's disease identifies new risk loci and implicates Abeta, tau, immunity and lipid processing. Nat Genet 51(3):414-430. https://doi.org/10.1038/s41588-019-0358-2

8. Jansen IE, Savage JE, Watanabe K et al (2019) Genome-wide meta-analysis identifies new loci and functional pathways influencing Alzheimer's disease risk. Nat Genet 51(3):404-413. https ://doi.org/10.1038/s41588-018-0311-9

9. Liyanage UE, Law MH, Ong JS et al (2018) Polyunsaturated fatty acids and risk of melanoma: a Mendelian randomisation analysis. Int J Cancer 143(3):508-514. https://doi.org/10.1002/ijc.31334

10. Yamagishi K, Nettleton JA, Folsom AR (2008) Plasma fatty acid composition and incident heart failure in middle-aged adults: the Atherosclerosis Risk in Communities (ARIC) Study. Am Heart J 156(5):965-974. https://doi.org/10.1016/j.ahj.2008.06.017 